\title{
The oblique effect in a mirror-tracing task
}

\author{
J. TIMOTHY PETERSIK \\ Ripon College, Ripon, Wisconsin 54971 \\ and \\ ALLAN J. PANTLE \\ Miami University, Oxford, Ohio 45606
}

\begin{abstract}
In a mirror-tracing task, 53 subjects were tested with vertically, horizontally, and obliquely oriented stimuli (narrow rectangles). Number of errors and time to completion were the dependent measures recorded. Following a 10-min practice period, the subjects were retested with the same stimuli. Results showed that subjects performed less efficiently with the oblique stimuli, in terms of both errors and time. Following practice, greater improvement was shown with the oblique stimuli, although for the temporal measure it still did not equal the performance with the horizontal and vertical stimuli. The tracings of the subjects gave an appearance that suggested that they made inefficient use of reafferent feedback when attempting to trace the oblique stimuli. A few subjects were immune to this perceptual-motor oblique effect.
\end{abstract}

The "oblique effect" has been characterized by Appelle (1972) as "a small but consistent superiority in performance when visual stimuli are horizontal or vertical, as opposed to oblique" (p. 266). The oblique effect manifests itself perceptually in lowered acuity, reduced contrast sensitivity, reduced evoked potential (see Appelle, 1972, for a review of this literature), and reduced increment sensitivity (Rentschler \& Fiorentini, 1974) in response to oblique stimuli. The effect is also evident in several reported perceptual-motor tasks (Appelle, 1972). These behavioral studies are paralleled by evidence that cortical "orientation detectors" are less narrowly tuned to oblique lines than to vertical and horizontal lines (Rose \& Blakemore, 1974).

One of us (Pantle) has informally demonstrated that linear oblique stimuli are more difficult to trace when viewed in a mirror than are horizontal and vertical stimuli. Because this effect has never been studied quantitatively, in the present paper we report a large and consistent superiority of vertical and horizontal stimuli over oblique stimuli in a simple mirror-tracing task. The effect is easily demonstrated and can make a useful classroom introduction to the oblique effect.

\section{METHOD}

\section{Subjects}

Subjects were 53 students enrolled in three sections of the general psychology laboratory course at Ripon College. Their ages ranged from 17 to 21 years. There was an approximately even ratio of males to females.

\section{Stimuli and Apparatus}

A set of two horizontal and two vertical (HV) rectangles was prepared and mimeographed on sheets of paper measuring $8.5 \times 11.0$ in. A similar set of two $45-\mathrm{deg}$ and two $135 \mathrm{-deg}$ oblique $(\mathrm{OBL})$ rectangles was also prepared and mimeographed.
For each set of stimuli, the rectangles measured $1.5 \times 13 \mathrm{~cm}$. A starting marker (an "X") was positioned at one end of each rectangle. The starting positions were located so that the subject would make one trace in each of four possible directions. Figure 1 shows the configuration of these stimuli.

During testing and practice, a stimulus set was placed on a standard laboratory mirror-tracing apparatus.

\section{Procedure}

By random determination, half the subjects began testing with the HV stimuli and half with the OBLs. While observing the stimuli and his pencil in the mirror only, the subject's task was to trace through the interiors of the four stimuli, from each starting point to the opposite end, as rapidly as possible without crossing the solid borders of the rectangles. If the subject did touch the border of a figure, he was to replace his pencil at the point of crossing and continue tracing. The order in which the four rectangles were traced was randomly determined for each subject. Immediately following completion of the first set of stimuli, the subject was tested with the alternate set (either HVs or OBLs).

Following the initial test, each subject used the eraser end of his pencil to practice tracing both HV and OBL stimuli for a total of $10 \mathrm{~min}$. Immediately after this practice session, each subject was again tested with new sets of $\mathrm{HV}$ and OBL stimuli, again in random order.

Subjects were timed on all tests, and an error was counted whenever the subject touched or crossed the border of a rectangle.

\section{RESULTS}

Figure 1 shows the tracings recorded by a typical subject during the initial test. As can be seen, this subject had little difficulty tracing the HV stimuli. On the other hand, he had difficulty even initiating appropriate movements when attempting to trace the OBL stimuli. Furthermore, his movements were not smooth and straight in the way that they were with the HV rectangles. Rather, at least with his first few trials, he made 
jerky back-and-forth movements in an attempt to progress forward. As can be seen in Figure 1b, this subject showed an improvement in performance during the tracing of the four OBL stimuli, although his performance with the OBL stimuli never matched his performance with the HV stimuli.

The results of this experiment are shown in Table 1. As can be seen in the first two columns and rows, performance with the HV stimuli was superior on both measures (time is measured in seconds), both during the initial test and after practice. Furthermore, practice seemed to lead to improvement in performance with both the HV and OBL stimuli.

In order to assess the difference in performance between the OBL and HV stimuli and to assess the effect of practice on these performances, separate repeated-measures analyses of variance were conducted on the time and error data. (We could find no satisfactory derived measure to reflect both time and errors on individual trials; e.g., the number of errors per second was inadequate because a subject could minimize such a score with the OBL stimuli by proceeding extremely slowly, and yet the time required for errorfree performance would be indicative of high task
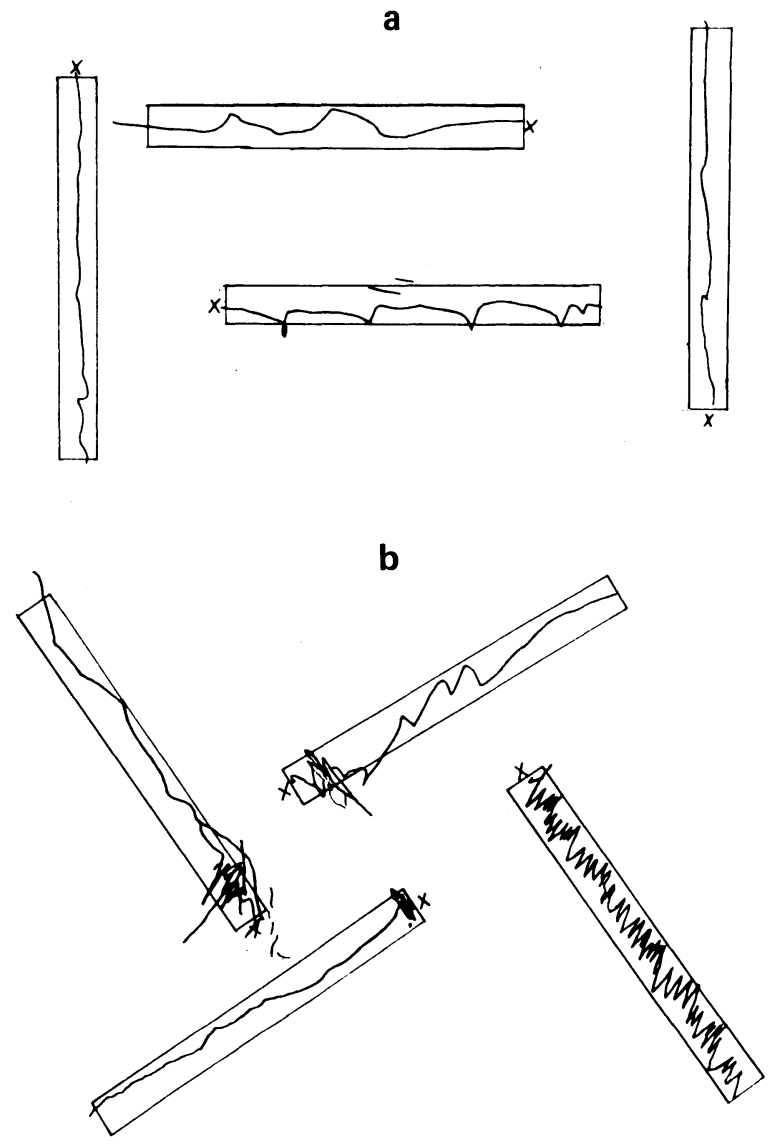

Figure 1. (a) Prepractice tracings of a typical subject working with the HV stimuli. (b) Prepractice tracings of the same subject with the OBL stimuli.
Table 1

Time (T) to Complete Tracings (in Seconds) and Number of Errors (E) Committed With Oblique and Horizontal-Vertical Stimuli as a Function of the Stage of Practice

\begin{tabular}{|c|c|c|c|c|c|c|}
\hline \multirow{3}{*}{$\begin{array}{l}\text { Stage of } \\
\text { Practice }\end{array}$} & \multicolumn{4}{|c|}{ Stimulus Type } & & \\
\hline & \multicolumn{2}{|c|}{ Oblique } & \multicolumn{2}{|c|}{$\mathrm{H}-\mathrm{V}$} & \multicolumn{2}{|c|}{ Difference } \\
\hline & $\mathrm{T}$ & $\mathrm{E}$ & $\mathrm{T}$ & E & $\mathrm{T}$ & $\mathrm{E}$ \\
\hline $\begin{array}{l}\text { Without } \\
\text { With } \\
\text { Improvement }\end{array}$ & $\begin{array}{c}169.42 \\
50.81 \\
118.61 *\end{array}$ & $\begin{array}{l}9.13 \\
2.47 \\
6.66 *\end{array}$ & $\begin{array}{l}34.68 \\
20.21 \\
14.47 \dagger\end{array}$ & $\begin{array}{r}1.13 \\
.81 \\
.32\end{array}$ & $\begin{array}{r}134.73 \dagger \\
30.60^{*}\end{array}$ & $\begin{array}{l}8.00 \dagger \\
1.66\end{array}$ \\
\hline
\end{tabular}

${ }^{*} p<.01 . \quad t p<.001$.

difficulty.) The analysis revealed a significant Orientation by Practice interaction both for errors $[\mathrm{F}(1,104)=8.96$, $p<.01]$ and for time required to complete the tracing $[F(1,104)=10.69, p<.01]$. Therefore, we analyzed the simple main effects of both orientation and practice for both measures. The third column of Table 1 shows the difference in both time and errors between the OBL and HV stimuli at both stages of practice. For the initial test, performance with the HV stimuli was superior both in terms of the number of errors committed $[F(1,52)=16.39, p<.001]$ and in terms of time required to trace $[F(1,52)=19.86, p<.001]$. After practice, however, performance with the HV stimuli was superior only with regard to the time required to complete the tracing $[\mathrm{F}(1,52)=9.33, \mathrm{p}<.01$; for errors, $F(1,52)=2.38, \mathrm{p}>.05]$.

The third row of Table 1 shows the improvement in performance as a function of practice for both the OBL and HV stimuli. The reduction in time required to complete the tracings was significant for both the OBL $[F(1,52)=16.16, p<.01]$ and $\mathrm{HV}[\mathrm{F}(1,52)=26.94$, $\mathrm{p}<.001]$ stimuli. However, the reduction in the number of errors committed was significant only for the OBL stimuli $[F(1,52)=9.36, \mathrm{p}<.01$; for the $\mathrm{HV}$ stimuli, $\mathrm{F}(1,52)=1.00, \mathrm{p}>.05]$.

\section{DISCUSSION}

The present experiment has shown the perceptual-motor consequences of the oblique effect to be especially potent in a mirror-tracing task. This is especially true in the initial attempts of naive subjects to trace oblique stimuli; however, such performance improves significantly with practice. Nevertheless, whereas most subjects were able to reduce the number of errors committed with the OBL stimuli after practice, they continued to require a greater period of time to conduct the tracing with the OBL stimuli than with the HV stimuli. Subjects did not show an improvement in the number of errors committed with the HV stimuli as a function of practice. This is no doubt due to the fact that subjects performed with a high degree of efficiency with these stimuli even before practice, so that little improvement was possible.

The mirror-tracing oblique effect makes an effective classroom demonstration and reveals a number of ancillary phenomena. For example, while attempting to trace the OBL stimuli, many of our subjects reported a feeling of a lack of voluntary control in producing movements in the desired direction. Such reports were uncommon with the HV stimuli. Furthermore, many of our subjects found it initially easier to progress through the oblique rectangles by making a number of "back-and-forth" 
movements. Both from subjective reports and from the appearance of the tracings, these efforts seem to be short ballistic movements. These findings suggest that while initially tracing the oblique stimuli, the typical subject makes an inefficient use of reafferent signals, with a subsequent inability to make compensatory movements.

A small percentage of our subjects seemed to be immune to this perceptual-motor oblique effect. They performed equally well with both HV and OBL stimuli and showed little improvement in performance with either as a function of practice. The nature of the differences between these "immune" subjects and the vast majority of subjects should be an important topic of future research.

\section{REFERENCES}

Appelle, S. Perception and discrimination as a function of stimulus orientation: The oblique effect in man and animals. Psychological Bulletin, 1972, 78, 266-278.

Rentschler, I., \& Fiorentini, A. Meridional anisotropy of psychophysical spatial interactions. Vision Research, 1974, 14, 1467 1473.

Rose, D., \& Blakemore, C. An analysis of orientation selectivity in the cat's visual cortex. Experimental Brain Research, 1974, 20, 1-17.

(Received for publication June 19, 1982.) 